\title{
Quantitative Stage Mapping of a Zircon grain by WDS on an SEM.
}

\author{
Stephen M. Seddio ${ }^{1}$ and Rita C. Economos ${ }^{2}$ \\ 1. Thermo Fisher Scientific, Fitchburg, WI, USA. \\ 2. Department of Earth Sciences, Southern Methodist University, Dallas, TX, USA.
}

Because of the effects of Bragg defocusing, accurate quantitative analysis by WDS can only be done with the electron beam in "spot mode" and with samples at the proper analytical working distance. As a result, WDS X-ray mapping in the SEM has only achieved simple raw counts mapping of a single element by rastering the beam over the sample. More complicated WDS X-ray mapping (quantitative or otherwise) has been relegated to the electron microprobe (e.g., [1]). However, modern SEMs and WDS systems permit quantitative WDS mapping in which a complete WDS quantitative analysis, including background and $\Phi(\rho z)$ corrections, is done at each pixel. Here, we use WDS quantitative analysis to map the concentration of $\mathrm{Hf}$ in a zoned zircon grain. Zircon has emerged as the most critical geochronological tool in the earth sciences. These tiny crystals are truly zircon-halfnon solid solutions and typically contain 10's of thousands of ppm Hf. Zr/Hf ratios are used as an index of magma evolution and Hf isotopes in zircon are an important recent tool employed to explore magma sources and processes. The ability to map Hf concentrations in detail and to pair this data with geochronology and Hf isotopes will yield important insights into the interpretation of zircon geochronology and the magmas from which zircons crystallize.

Quantitative WDS maps were acquired using a Thermo Scientific ${ }^{\text {TM }}$ MagnaRay ${ }^{\text {TM }}$ Parallel beam WDS spectrometer and a JEOL JSM-7001F FE-SEM. Data were processed using the Thermo Scientific ${ }^{\mathrm{TM}}$ NORAN ${ }^{\mathrm{TM}}$ System 7 microanalysis system. The quantitative WDS map was acquired by automatically slewing the SEM stage over a $106 \times 46$ grid while keeping the beam at a fixed position. The resulting map is $106 \times 46$ pixels with a $2 \mu \mathrm{m}$ resolution. All measurements were made using a $15 \mathrm{kV}$ beam accelerating voltage and a focused electron beam that was set to $206 \mathrm{nA}$ at the beginning of the run. The beam current was measured at the beginning of each analysis.

Standardization was done using a Hf-bearing zirconia (for $\mathrm{Hf} \mathrm{M} \alpha$ and $\mathrm{Zr} \mathrm{L} \alpha$ ) and quartz (for Si $\mathrm{K} \alpha$ ) on a commercially prepared (SPI), carbon coated mineral standards mount. The $\mathrm{O}$ concentration for each analysis was calculated by stoichiometry.

The zircon sample was picked from a granite from the Cretaceous Cadiz Valley Batholith in the central Mojave Desert. This grain was mounted in epoxy, polished, and carbon coated. SIMS geochronology and spot trace element analyses were previously conducted on two spots on the grain (Economos, pers. comm.) constraining absolute $\mathrm{Hf}$ concentrations and indicating that there are trace or minor concentrations of the REEs, Th, and $\mathrm{U}$, which were not included in these analyses and explain the resulting somewhat low analytical totals.

For unknown and standard analyses, Si K $\alpha$ and $\mathrm{Zr}$ L $\alpha$ were each counted on-peak for $5 \mathrm{~s}$ and off-peak for $2.5 \mathrm{~s}$ (at both low and high positions). Hf $\mathrm{M} \alpha$ was counted until the error (background corrected) from counting statistics was better than $2 \%$, which was typically achieved after $\sim 11 \mathrm{~s}$ on-peak. Off-peak measurement positions were confirmed to be free of higher order reflections or trace element peaks by inspection of WDS energy scans. 
4,876 analyses were acquired, from which $\mathrm{Zr}$, Si, Hf, (Fig. 1) and O quantitative concentrations maps were extracted. The maps reveal that the zircon grain is strongly zoned with respect to the $\mathrm{Hf}$ concentration.

The full range of WDS mapping (raw counts, net counts, and quantitative) is now available to the SEM user. Additionally, an EDS spectrum could be concurrently acquired with the WDS measurements, meaning that any arrangement of EDS or WDS standards-based, quantitative stage maps could be extracted. For example, when mapping zircon, $\mathrm{Zr}$ and Si could be mapped with EDS and trace elements (e.g., Hf) could be mapped with WDS.

\section{References:}

[1] PK Carpenter, SN North, BL Jolliff, and JJ Donovan, Lunar Planet. Sci. Conf. 44 (2013) p. 1827.

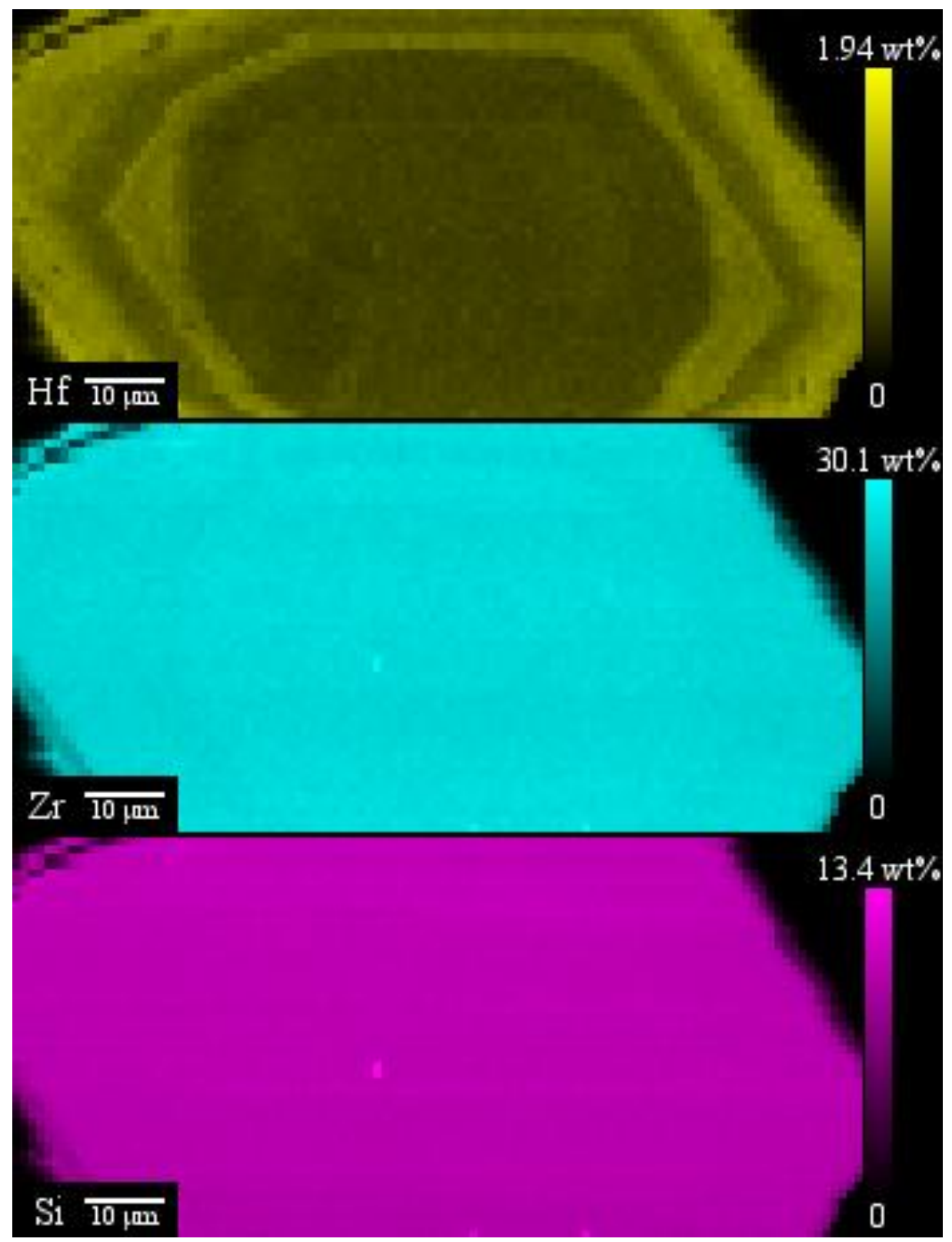

Fig. 1. Hf, Zr, and Si quantitative elemental maps of a zircon grain (see text for provenance). Maps are $106 \times 46$ pixels with a resolution of $2 \mu \mathrm{m}$ per pixel. 